\title{
Yes, Indeed, Idiosyncratic Risk Matters for Socially Responsible Investments!
}

\author{
Li Huimin \\ Department of Accounting, Finance and Economics \\ Griffith University, Australia \\ E-mail: jane.li@griffith.edu.au \\ Tel: +61439785461 \\ Cheung Adrian \\ Department of Accounting, Finance and Economics \\ Griffith University, Australia \\ E-mail: a.cheung@griffith.edu.au \\ Tel: +6173735 7644 \\ Roca Eduardo \\ Department of Accounting, Finance and Economics \\ Griffith University, Australia \\ E-mail: e.roca@griffith.edu.au \\ Tel: +61737357583
}

\begin{abstract}
We provide empirical evidence regarding the effect of stock market regimes on Social Responsible Investment (SRI). Using the Markov Switching Model, we identify three market regimes for the study period between June 2001 and December 2009 in the US. These regimes are the low, medium, and high volatility states. We find a positive relationship between the idiosyncratic risk (i.e. unsystematic risk) and return during low and medium volatility states. However, this positive relationship tends to disappear during high volatility states. In addition, our analysis suggests that idiosyncratic risk has no forecasting power over SRI future returns. Overall, our findings imply that SRI investors are rewarded for bearing the additional SRI specific risk (idiosyncratic risk) when the market is less volatile. This reward, however, becomes uncertain during periods of high market volatility.
\end{abstract}

Keywords: Idiosyncratic Risk, Unsystematic Risk, Socially Responsible Investment, Stock Market Regimes

\section{Introduction}

Socially Responsible Investment (SRI) portfolios suffer from under-diversification due to the additional screening that they employ, and this has very important implications in terms of idiosyncratic risks (also called unsystematic risk) (Aaker and Jacobson, 1987; Carroll and Wei, 1988; Galema, Plantinga and Scholtens, 2008). However, to the best of our knowledge no study has investigated the relationship between SRI idiosyncratic risk and return. This paper sheds light on this 
issue in regard to the SRI literature. Goyal and Santa-Clara (2003) argued that "idiosyncratic risk matters!" In this paper we confirm that indeed it does, particularly for socially responsible investments.

The lack of sufficient studies on the SRI unsystematic risk can be associated with at least two reasons. First, different companies may have different risk exposures based on the extent to which they are involved in SRI activities. Therefore, it is difficult to precisely gauge the involvement of these companies in SRI. Second, SRI portfolios usually employ different screening standards and ranking criteria, which make their exposure hard to estimate. These difficulties make measuring the unsystematic risk a difficult matter. As a result, previous papers have investigated the total risk premium, which is simple and straightforward to calculate. However, studying the total risk premium rather than the unsystematic risk premium is underprivileged due to the possibility of misinterpreting the impact of the role of risk on returns both empirically and conceptually (Aaker and Jacobson, 1987). We overcome this problem by adopting the unsystematic risk measures from early studies of under-diversified portfolios (see Cheung and Wang, 1992; Angelidis and Tessaromatis, 2008; Bali and Cakici, 2008).

Generally, investors holding investments with larger unsystematic risk will require a higher return to compensate for the imperfect diversification (Merton, 1987). However, the existing literature offers contradicting empirical results over the stock market unsystematic risk premium. For instance, Aaker and Jacobson (1987), Cox and Griepentrog (1988), Barber (1994), and Campbell, et al. (2001) found a significant positive unsystematic risk premium, while Cheung and Wang (1992), Bali and Cakici (2008,) and Bollen, Skotnicki and Veeraraghaven (2009) have reported no significant relationship between the unsystematic risk and return in the US, Australia, and Hong Kong markets respectively. These studies have all applied a single-period regression approach, which is simple, intuitive, and widely used, but ineffective in estimating the unsystematic risk premium (Fu, 2005; Jiang and Lee, 2006; Carroll and Wei, 1988; Bali, Cakici, Yan, and Zhang, 2005). The criticism of the single-period study has enhanced the popularity of multiple-period studies. However, the findings of multiple-period studies are still inconclusive. For example, Carroll and Wei (1988) contended that unsystematic risk premium only exists in January, but not in the rest of the year. Bali et al. (2005) asserted that unsystematic risk was rewarded in the US over the period 1963 to 1999, but not for the period 1963 to 2001. Tang and Shum $(2006 ; 2007)$ also provided empirical evidence of stock market conditions as a notable determination for the value of the unsystematic risk premium to explain the time varying effect on this issue. Tang and Shum found a significant positive unsystematic risk premium during the up markets in both 2006 and 2007. However, in relation to the down markets, they found a significant negative risk premium in 2006 but no rewards for unsystematic risk in 2007.

Although the non-linearity of unsystematic risk premium has been recognized, multiple-period studies failed to precisely determine the stock market regimes. First, these studies mostly predetermine the breakpoints of stock market regimes into limited periods. Second, the traditional linear model does not allow for the possibility of the unsystematic risk-return relationship change within each study period (Angelidis and Tessaromatis, 2009). Also, using pre-determined breakpoints of the market may not allow precise identification of market conditions for accurate findings, since market conditions change rapidly and unpredictably ( $\mathrm{Fu}, 2005)$. To overcome this problem, Angelidis and Tessaromatis (2009) have applied the Markov Switching (MS) model to forecast the relationship between unsystematic risk and the UK stock market returns. They found that the significant positive relationship is only valid during the low volatility regime, and it disappears during the high volatility regime. Our study aims particularly to uncover the changes of the unsystematic risk and the changes of the unsystematic risk-return relationship on the SRI indices across different market regimes by applying the MS model. Also, we intend to examine the forecasting power of unsystematic risk over SRI return. 
We find that SRI unsystematic risk increases when market volatility rises. We also find that SRI does indeed reward investors during low and medium volatility states in terms of the social screens (the unsystematic risk), but not during high volatility states. This empirical evidence provides important implications for SRI-investors and corporations, especially for risk-averse investors.

The remainder of the paper is organised as follows. In Section 2 we explain our data collection process. Then, we describe our methodology in Section 3. Section 4 provides our empirical results. Finally, Section 5 contains concluding remarks.

\section{Research Data}

We investigate seven equity indices from the most well developed US SRI market. These indices have been constructed and published by three different suppliers. They are the well known SRI index families KLD Research and Analytics Inc. (KLD), Calvert, and FTSE. For the purpose of examining the SRI risk return relationship, studying indices has significant advantages over studying funds. For example, the SRI effect can be measured without being confounded by other issues such as the specific screening aims and objectives, the transaction costs, and the funds managers' timing skills and stock-picking skills (Schroder, 2007; Statman and Glushkov, 2009).

The seven SRI indices have different capitalization sizes. Included are three large caps, two mixed caps, one medium cap, and one small cap. Panel A of Table 1 presents the seven SRI indices, the benchmark indices, the abbreviation, and the SRI launching date. Each SRI index is benchmarked by a closely approximated conventional index based on their investment universe. Panel B of Table 1 presents the investment universe for the SRI indices and their benchmark indices.

Table 1: Characteristics of SRI and Benchmark Indices

\begin{tabular}{|c|c|c|c|c|c|c|c|}
\hline \multicolumn{8}{|l|}{ Panel A } \\
\hline \multicolumn{2}{|c|}{ Index Name } & Abbrev & Launch Date & \multicolumn{3}{|c|}{ Benchmark } & Abbrev. of Benchmark \\
\hline \multicolumn{2}{|c|}{ FTSE4Good US } & FUS & $7 / 2001$ & \multicolumn{3}{|c|}{ FTSE Local USA } & FTSE \\
\hline \multicolumn{2}{|c|}{ KLD Domini 400 Social Index } & DSI & $5 / 1990$ & \multicolumn{3}{|c|}{ S and P 500} & SandP \\
\hline \multicolumn{2}{|c|}{ KLD Broad Market Social Index } & USSA & $1 / 2001$ & \multicolumn{3}{|c|}{ Russell 3000} & R3000 \\
\hline \multicolumn{2}{|c|}{ KLD large Cap Social Index } & USSL & $1 / 2001$ & \multicolumn{3}{|c|}{ Russell 1000} & R1000 \\
\hline \multicolumn{2}{|c|}{ Calvert Social Index } & CAL & $6 / 2000$ & \multicolumn{3}{|c|}{ Russell 1000} & $\mathrm{R} 1000$ \\
\hline \multicolumn{2}{|c|}{ KLD Small Cap Social Index } & USSS & $1 / 2001$ & & \multicolumn{2}{|c|}{ Russell 2000} & R2000 \\
\hline \multicolumn{2}{|c|}{ KLD Mid Cap Social Index } & USSM & $1 / 2001$ & & and P Mid Cap 4 & Index & SandPM \\
\hline \multicolumn{8}{|c|}{ Panel B: Indices Investment Universe } \\
\hline Index & \multicolumn{3}{|c|}{ Index Invest Universe } & & Benchmark & \multicolumn{2}{|c|}{ Benchmark Invest Universe } \\
\hline DSI & \multicolumn{4}{|c|}{250 SandP and 100 Large and Mid cap, 50 small cap } & SandP & \multicolumn{2}{|c|}{500 large cap US equities } \\
\hline CAL & \multicolumn{3}{|c|}{1000 Largest US Equities } & & $\mathrm{R} 1000$ & \multicolumn{2}{|c|}{1000 Largest US Equities } \\
\hline USSL & \multicolumn{3}{|c|}{400 Largest US Equities } & & $\mathrm{R} 1000$ & \multicolumn{2}{|c|}{1000 Largest US Equities } \\
\hline FUS & \multicolumn{3}{|c|}{100 Largest Equities } & & FTSE & \multicolumn{2}{|c|}{100 Largest Equities } \\
\hline USSA & \multicolumn{3}{|c|}{3000 Largest US Equities } & & R3000 & \multicolumn{2}{|c|}{3000 Largest US Equities } \\
\hline USSM & \multirow{2}{*}{\multicolumn{3}{|c|}{600 Mid-Cap US equities }} & & SandPM & \multirow{2}{*}{\multicolumn{2}{|c|}{$\begin{array}{l}\text { Medium-sized } 400 \text { US Equities } \\
2000 \text { Small Cap US Equities }\end{array}$}} \\
\hline USSS & & & & & R2000 & & \\
\hline
\end{tabular}

We choose the study period from June 2001 to December 2009 to make sure the time interval is long enough to include as many US SRI indices with full data history as possible. Also, we aim to include part of the recession that occurred from March 2001 to October 2001, and the one that began in December 2007. Including these two recessions allows more opportunities for testing the variation of SRI indices performance during different market volatility conditions, since most recessions overlap market downturns (Gonzalez et al., 2006). 
Our main data source is the suppliers of the indices. We also use the database of Thomson Reuters Tick History (TRTH) to collect some of the index series. We calculate the monthly returns based on the discrete return formula $\mathrm{R}_{\mathrm{t}}=\left(\mathrm{P}_{\mathrm{t}}-\mathrm{P}_{\mathrm{t}-1}\right) / \mathrm{P}_{\mathrm{t}-1} \times 100_{1}$

\section{Research Methodology}

We apply the Markov Switching (MS) model to decompose the time series into a finite sequence of regimes. Each of the processes is linear, but their combination is non-linear. This creates a combination of variables with a model, which describes the stochastic process that determines the switch from one regime to another one by means of the Markov Chain $\left[^{2}\right]$. A Markov Chain has the probability of $\mathrm{P}_{\mathrm{ij}}$ which can be defined as:

$$
\mathrm{p}_{\mathrm{ij}}=\mathrm{p}_{\mathrm{r}}\left(\mathrm{S}_{\mathrm{t}-1}=\mathrm{i} \mid \mathrm{S}_{\mathrm{t}}=\mathrm{j}\right), \sum_{\mathrm{j}=1}^{\mathrm{M}} \mathrm{p}_{\mathrm{ij}}=1 \forall \mathrm{i}, \mathrm{j} \in\{1, \ldots, M\} \text {. }
$$

$P_{i j}$ indicates that the probability for a variable $S_{t}$ in state $i$ is followed by state $j$. Therefore, $\mathrm{P}_{\mathrm{i} 1}+\mathrm{P}_{\mathrm{i} 2}+\mathrm{P}_{\mathrm{i} 3}+\ldots+\mathrm{P}_{\text {in }}=1$. A transition matrix $\mathrm{p}$ produced from the stochastic process can be computed as follows:

$$
\mathrm{p}=\left[\begin{array}{cccc}
\mathrm{P}_{11} & \mathrm{P}_{12} & \ldots & \mathrm{P}_{1 \mathrm{n}} \\
\mathrm{P}_{21} & \mathrm{P}_{22} & \ldots & \mathrm{P}_{2 \mathrm{n}} \\
\ldots & \ldots & \ldots & \ldots \\
\mathrm{P}_{\mathrm{n} 1} & \mathrm{P}_{\mathrm{n} 2} & \ldots & \mathrm{P}_{\mathrm{nn}}
\end{array}\right]
$$

Each regime is the realization of a first-order Markov chain, and the state $S_{t}$ is unobservable and we will treat it as a latent variable. Following the studies of Krolzig (1997), Roca and Wong (2008), and Guidolin and Hyde (2009), we estimate the regression model underlying the MS model as:

$$
\mathrm{Y}_{\mathrm{t}}=\mathrm{v}_{\left(\mathrm{s}_{\mathrm{t}}\right)}+\sum_{\mathrm{k}=1}^{\mathrm{p}} \beta_{\mathrm{k}\left(\mathrm{s}_{\mathrm{t}}\right)} \cdot \mathrm{X}_{\mathrm{t}-\mathrm{k}}+\sum_{\mathrm{j}=1}^{6} \alpha \cdot \mathrm{Z}_{\mathrm{t}}+\mu_{s_{t}}
$$

Where, $Y_{t}=\left[R_{j t}-R_{f t}\right]$, i =index $i$, $v$ is a vector of intercepts in state $s_{t}, \beta_{k}$ is the coefficient of market risk premium $X_{t}=\left[R_{m t}-R_{f t}\right]$ at lag k. $Z_{t}=\left[R_{j t}-R_{f t}\right] j \neq i, k$ stands for the lag length, $\alpha$ is the coefficient parameters for SRI indices other than index $i$, and $\mu_{s_{t}} \sim N\left(0, \sum S_{t}\right)$ is the vector of return derived from the unsystematic risk that is assumed to be jointly normal distributed with zero mean and state-specific covariance matrix at state $\mathrm{t}$.

To be precise, we can rewrite this general model into seven different regression forms, based on the choice of the dependent variable of the SRI index. For example, we can form the regression model for index DSI as follows:

$$
\begin{aligned}
& \mathrm{R}_{\mathrm{DSIt}}-\mathrm{R}_{\mathrm{ft}}=\mathrm{v}_{\left(\mathrm{s}_{\mathrm{t}}\right)}+\sum_{\mathrm{j}=1}^{\mathrm{q}} \beta_{\mathrm{j}, \mathrm{s}_{\mathrm{t}}}\left[\left(\mathrm{R}_{\mathrm{S} \& \mathrm{P}}-\mathrm{R}_{\mathrm{f}}\right)_{\mathrm{t}-\mathrm{j}}\right]+\alpha_{1} \cdot\left(\mathrm{R}_{\mathrm{CAL}}-\mathrm{R}_{\mathrm{f}}\right)_{\mathrm{t}}+\alpha_{2} \cdot\left(\mathrm{R}_{\mathrm{USSA}}-\mathrm{R}_{\mathrm{f}}\right)_{\mathrm{t}}+ \\
& \alpha_{3} \cdot\left(\mathrm{R}_{\mathrm{USSL}}-\mathrm{R}_{\mathrm{f}}\right)_{\mathrm{t}}+\alpha_{4} \cdot\left(\mathrm{R}_{\mathrm{USSM}}-\mathrm{R}_{\mathrm{f}}\right)_{\mathrm{t}}+\alpha_{5} \cdot\left(\mathrm{R}_{\mathrm{USSS}}-\mathrm{R}_{\mathrm{f}}\right)_{\mathrm{t}}+\alpha_{6} \cdot\left(\mathrm{R}_{\mathrm{FUS}}-\mathrm{R}_{\mathrm{f}}\right)_{\mathrm{t}}+\varepsilon_{\mathrm{S}_{\mathrm{t}}}
\end{aligned}
$$

We adopt the returns of SRI indices other than the dependent variable index as control variables. This is because they have unspecified and unobservable common factors, which might affect the return of the dependent variable. Adding those indices returns as control variables, can effectively improve the regression explanatory power. Meanwhile, we exclude book-to-market ratio

Although using monthly data provides us only 102 observations for the MS model, our data are still reasonable for the MS model as compared to the studies of Guo, Brooks and Shami (2010), and Marco, Zacharias and Martin (2003), which have used 124 and 89 observations for the MS model respectively.

2 A Markov Chain is a system which switches among several states, with the next state depending only on the current state. 
(HML), the momentum (MOM), and the size ratio (BMS) as control variables of our model because we can capture the effects of HML, MOM, and BMS on the SRI return simply by comparing with their closely approximated benchmark index (Schroder (2007).

Once we have identified the market regimes for each SRI index, the next step is to measure the unsystematic risk in each regime and test the strength of the relationship between the unsystematic risk and return during each regime.

Following Angelidis and Tessaromatis (2008) and Bali and Cakici (2008), we apply the indirect decomposition method to measure the unsystematic risk. This method assumes the return of each SRI index is driven not only by market factors, but also the SRI specific factor $\varepsilon_{\mathrm{i}}$. First, we estimate the following regression model:

$$
\mathrm{R}_{\mathrm{i}, \mathrm{t}}-\mathrm{R}_{\mathrm{f}, \mathrm{t}}=\beta_{\mathrm{i}, \mathrm{t}} \cdot\left(\mathrm{R}_{\mathrm{m}, \mathrm{t}}-\mathrm{R}_{\mathrm{f}, \mathrm{t}}\right)+\varepsilon_{\mathrm{i}, \mathrm{t}}
$$

where, $R_{i, t}-R_{f, t}$ is the excess return of SRI index during month $t$, and $\left(R_{m, t}-R_{f, t}\right)$ is the benchmark index excess return during month t. After deriving regression residual $\left(\varepsilon_{i, t}\right)$ for each SRI index in each month of the study period, we then estimate the index's unsystematic risk on time $t=0$ based on the standard error of regression residuals from the preceding 12 months until the end of the study period (Cheung and Wang, 1992).

Afterwards, we apply the following regression model to estimate the relationship $v_{\mathrm{i}}$, between unsystematic risk and the SRI indices' excess returns in regime t.

$$
\mathrm{R}_{\mathrm{i}, \mathrm{t}}-\mathrm{R}_{\mathrm{f}, \mathrm{t}}=\mu+v_{\mathrm{i}, \mathrm{S}_{\mathrm{t}}} \cdot \mathrm{X}_{\mathrm{i}, \mathrm{t}}+\delta_{\mathrm{i}, \mathrm{t}}
$$

where, $R_{i, t}-R_{f, t}$ is the excess return of the SRI index, $\mu$ is the intercept, $X_{i, t}$ is the unsystematic risk derived from equation (3), and $\delta_{\mathrm{i}, \mathrm{t}}$ is the error term.

\section{Empirical Results}

\subsection{Diagnostic Test Results}

We apply the Augmented Dickey-Fuller (ADF) test to identify the order of integration of index excess return time series. The ADF test for the null hypothesis of non-stationary (unit root) against the alternative hypothesis of stationary (no unit root) is carried out in the original form with trend and intercept. We use a data-driven automatic lag length selection procedure in E-View to select the number of lagged difference terms (or lag length).

The ADF test rejects the null hypothesis of a unit root at $1 \%$ level of significance for all the index time series, since the ADF test t-statistics are all less than -7.3978, while the test critical value at 1 per cent is -4.0515 . Hence we conclude the excess returns of the SRI indices and their benchmarks are all stationary and no further differentiating or testing for co-integration is needed.

\subsection{Model Selection Process}

The next step is to decide the appropriate Markov Switching Process by finding the switching parameters, the proper number of regimes, and the appropriate lag length to use by applying the SIC test. The reason for using the SIC test other than the usual Likelihood ratio or the Akaike Information Criterion (AIC) is due to the introduction of a stronger penalty term for the number of parameters in the SIC criteria, which makes its application more efficient (Sylvain, 2009).

Now we apply a computationally intensive approach to run each regression 36 times by adjusting the number of lags and regimes and choosing the different switching parameters in use. By doing this, we are able to find the model with the lowest SIC value for each SRI index. We restrict the number of regimes and lags to three, since including more regimes and lags would pose extreme computational problems and make the number of parameters difficult to estimate. The parameters such as intercept term (I), beta coefficient $(\beta)$, and error term $(\mathrm{H})$ can be either state dependent 
(switching) or remain constant (non-switching). Table 2 shows the models selected for the individual SRI indices. Except DSI, which fits the model MSIH (3)-(3), the other six SRI indices fit the model MSH (3)-(2).

Table 2: $\quad$ MS Models for Each Index Groups

\begin{tabular}{|l|c|c|c|}
\hline Index & Benchmark & MSM & SIC \\
\hline DSI & SandP & MSIH(3)-(3) & 13.9715 \\
USSA & R3000 & MSH(3)-(2) & 11.6511 \\
USSL & R1000 & MSH(3)-(2) & 13.9186 \\
CAL & R1000 & MSH(3)-(2) & 13.9186 \\
USSS & R2000 & MSH(3)-(2) & 17.7568 \\
USSM & SandPM & MSH(3)-(2) & 15.8371 \\
FUS & FTSE & MSH(3)-(2) & 14.0393 \\
\hline
\end{tabular}

Notes: 1 . The first number in the brackets is the number of regimes, and the second one is the number of lags. In this study, all of the models have 3 regimes and 2 lags, except that DSI has 3 lags.

\subsection{Transition Probabilities and Characteristics of Market Regimes}

Table 3 presents the transition probabilities, the number of observations, duration, and Ergodic probability for the seven indices in each of the three identified regimes.

Table 3: Transition Probabilities and Characteristics of MS Models

\begin{tabular}{|l|l|c|c|c|c|c|c|}
\hline \multicolumn{9}{|c}{ Transition Probabilities: (of \%) } & \multirow{2}{*}{ Obs } & \multirow{2}{*}{ Duration } & $\begin{array}{c}\text { Ergodic } \\
\text { Prob. }\end{array}$ \\
\hline \multirow{3}{*}{ USSM } & Regime 1 & 83.52 & 9.83 & 6.66 & 61 & 6.07 & 60.90 \\
& Regime 2 & 24.80 & 61.99 & 13.22 & 17 & 2.63 & 18.17 \\
& Regime 3 & 26.44 & 4.41 & 69.15 & 22 & 3.24 & 20.93 \\
\hline \multirow{3}{*}{ USSL CAL } & Regime 1 & 91.91 & 1.64 & 6.45 & 62 & 12.36 & 62.10 \\
& Regime 2 & 16.20 & 51.39 & 32.41 & 19 & 2.06 & 18.98 \\
& Regime 3 & 10.29 & 43.40 & 46.31 & 19 & 1.86 & 18.92 \\
\hline \multirow{3}{*}{ USSS } & Regime 1 & 92.16 & 6.25 & 1.59 & 64 & 12.76 & 65.81 \\
& Regime 2 & 23.36 & 64.24 & 12.40 & 18 & 2.8 & 18.50 \\
& Regime 3 & 5.32 & 15.97 & 78.71 & 18 & 4.7 & 15.68 \\
\hline \multirow{3}{*}{ FSSA } & Regime 1 & 94.74 & 0.00 & 5.26 & 58 & 19.02 & 58.56 \\
& Regime 2 & 15.52 & 51.74 & 32.74 & 20 & 2.07 & 19.84 \\
& Regime 3 & 0.00 & 44.33 & 55.67 & 22 & 2.26 & 21.60 \\
\hline \multirow{3}{*}{ DSI } & Regime 1 & 96.52 & 1.724 & 1.754 & 58 & 28.75 & 58.54 \\
& Regime 2 & 10.29 & 46.31 & 43.40 & 20 & 1.86 & 19.78 \\
& Regime 3 & 0.00 & 44.33 & 55.67 & 22 & 2.26 & 21.68 \\
\hline
\end{tabular}

Notes: 1. Obs stands for the number of observations in a regime. Ergodic prob. stands for the ergodic probability, which detects the aperiodicity and the irreducibility of the regime for the Markov Chain.

As it can be seen, Regime 1 is the most persistent one with the longest duration and is characterized by low volatility and moderate return. It represents the state that no abnormal market boom occurred and shares are valued at their "fair price". This regime represents most of the study period extending from 2003 to 2007 . Regime 3 is corresponding to the highest volatility and the lowest return state. It coincides to the periods over which stock markets distress events occurred, such as the September 11 attacks in 2001, the Adelphia bankruptcy in 2002, the WorldCom bankruptcy in 2003, and the subprime crisis in 2008. Regime 2 on the other hand, maybe associate with the speculative bubbles or the government intervention. It is generally displayed by the disassociation between price and value (overvalued share price) and is characterized with a medium volatility and 
high return state. Both Regimes 2 and 3 fall between 2001 to 2003 and 2008 to 2009 and switch rapidly between each other, as illustrated in Figures 1 to 6 .

Figure 1: Probabilities of Regimes for the FUS
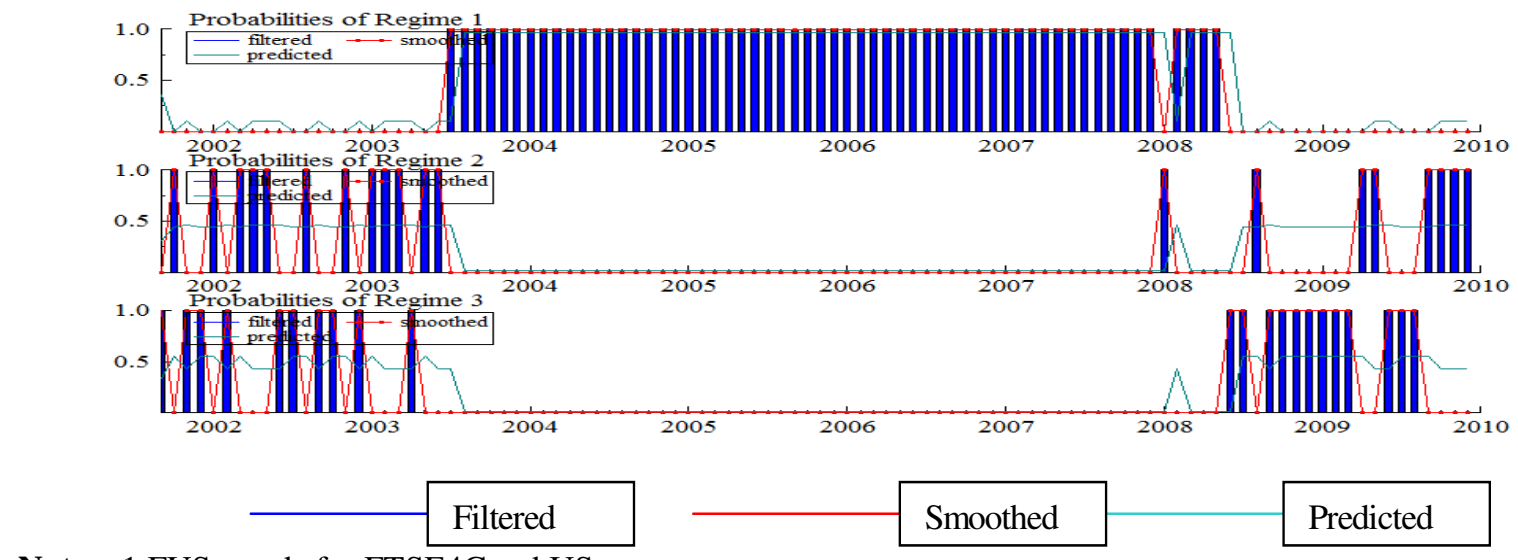

Notes: 1.FUS stands for FTSE4Good US.

Figure 2: Probabilities of Regimes for the USSS

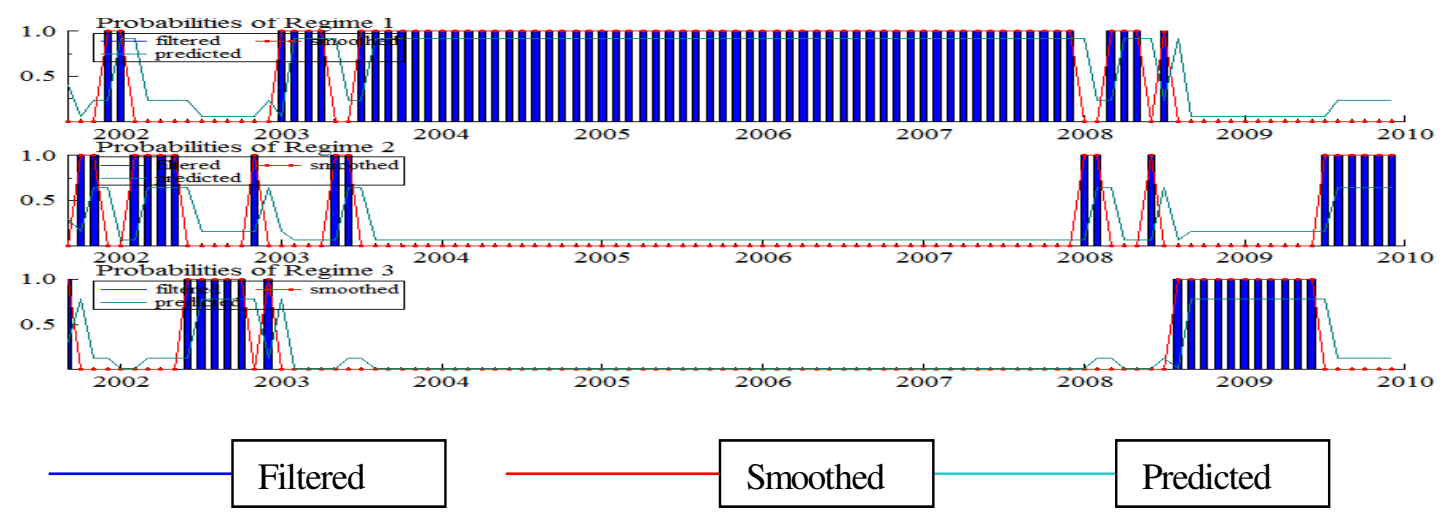

Notes: 1.USSS stands for KLD Small Cap Social Index.

Figure 3: Probabilities of Regimes for the DSI

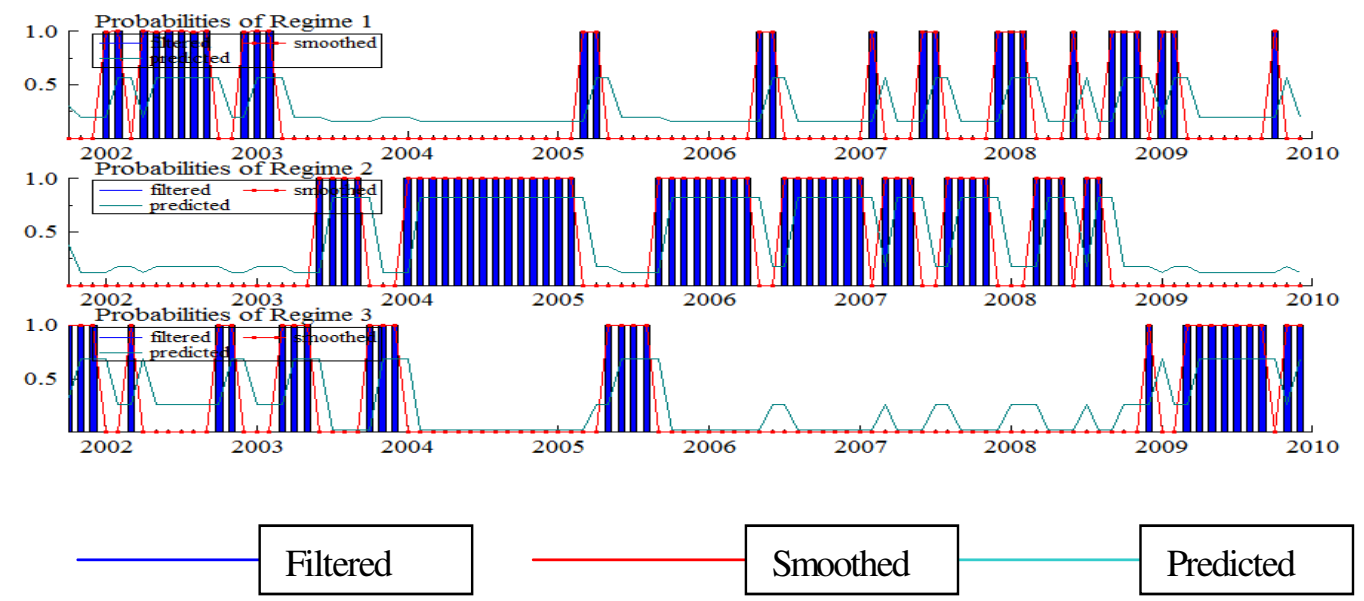

Notes: 1. DSI stands for KLD Domini 400 Social Index.

2. The Regime 2 denotes the stable state with moderate return and low volatility, the Regime 3 denotes high return and medium volatility, and Regime 1 represents the low return and high volatility state. Therefore, the regime numbers here are different from the ones in the text content. 
Figure 4: Probabilities of Regimes for the USSM

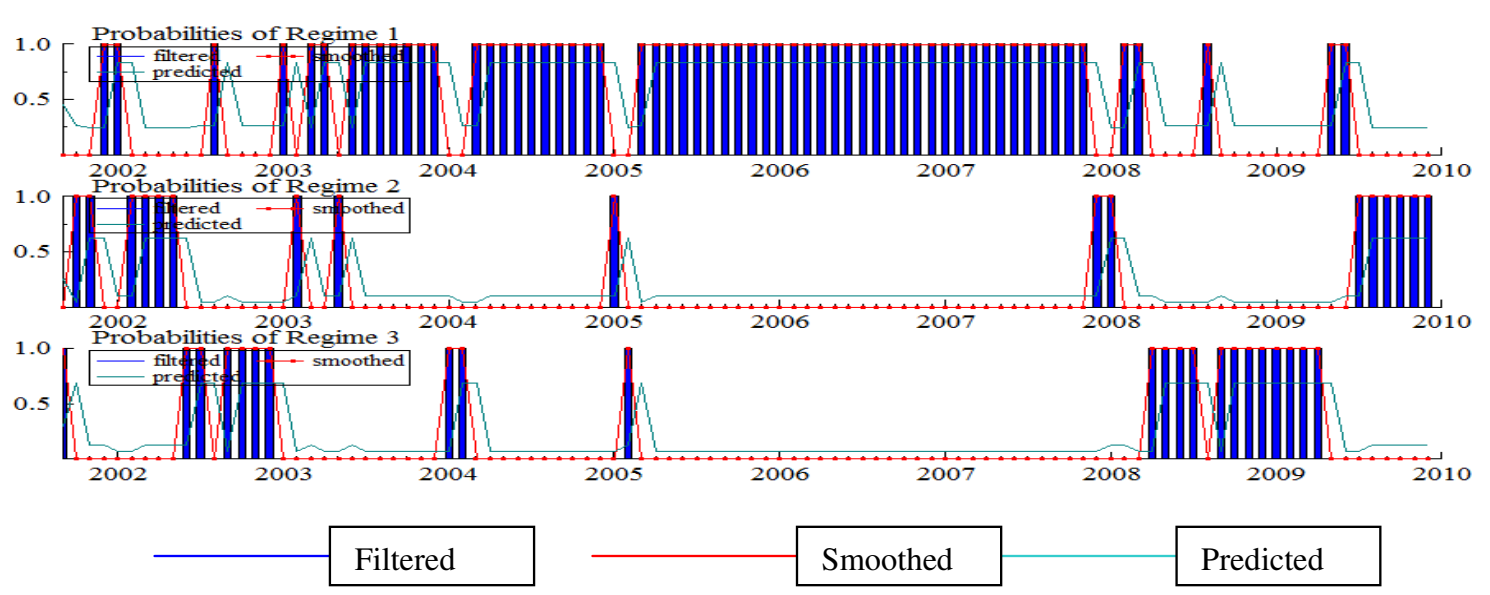

Notes: 1.USSM stands for KLD Mid Cap Social Index.

Figure 5: Probabilities of Regimes for the USSA
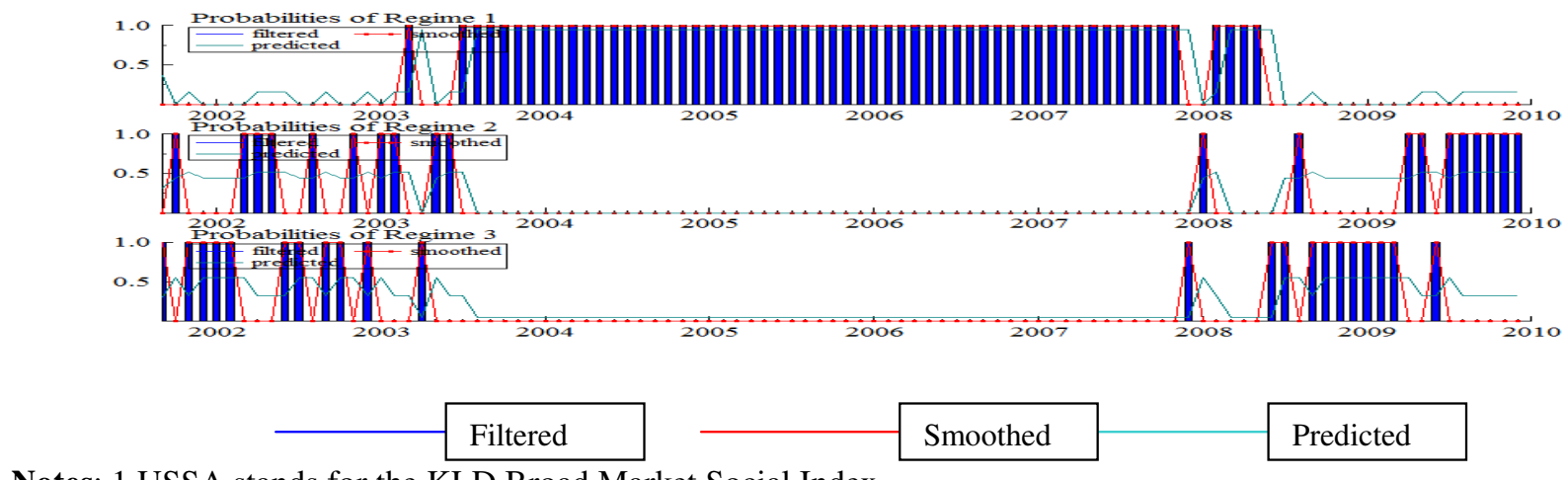

Notes: 1.USSA stands for the KLD Broad Market Social Index.

Figure 6: Probabilities of Regimes for the CAL and the USSL

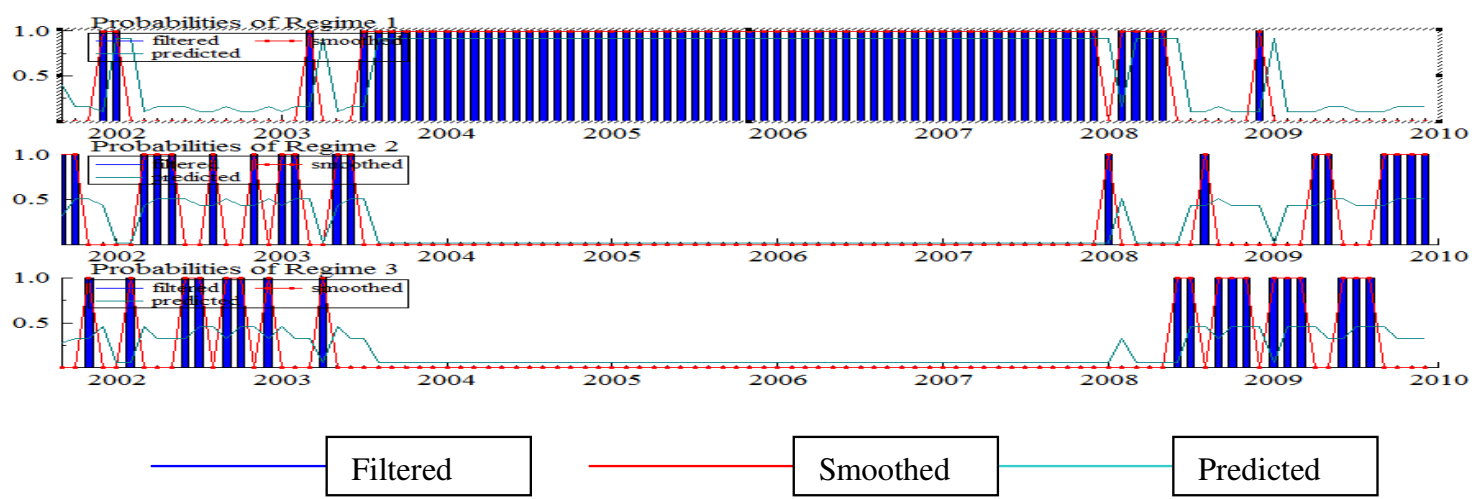

Notes: 1.CAL stands for the Carvel Social Index, and USSL stands for the KLD Large Cap Social Index.

\subsection{Unsystematic Risk (Idiosyncratic Risk)}

In Table 4, we present the unsystematic risk for each regime generated by the MS model. 
Table 4: $\quad$ Estimated Unsystematic Risk

\begin{tabular}{|l|l|l|c|c|c|c|c|}
\hline & DSI & CAL & USSA & USSL & USSM & USSS & FUS \\
\hline$\sigma_{1}$ & 1.88 & 2.66 & 2.74 & 2.60 & 2.96 & 3.37 & 3.32 \\
$\sigma_{2}$ & 3.13 & 4.60 & 4.45 & 4.59 & 5.95 & 5.58 & 3.47 \\
$\sigma_{3}$ & 2.29 & 8.88 & 8.49 & 8.49 & 8.30 & 9.76 & 9.93 \\
\hline
\end{tabular}

Notes: $1 . \sigma_{1}, \sigma_{2}$, and $\sigma_{3}$ stand for the standard error of residuals in Regime 1, 2, and 3 respectively.

The unsystematic risk for most of the SRI indices increases when the market moves from low volatility state (Regime 1) to medium volatility state (Regime 2 ), and then to high volatility state (Regime 3). This result indicates that the market cycles have an impact on the unsystematic risk in the same way as on the total risk. This is reasonable because the systematic risk remains constant during the three regimes for both MSIH and MSH models.

The question to ask is why SRI unsystematic risk is so highly regime-dependent. Possible explanations at the firm level are as follows. First, the SRI more on social objectives during low volatility states and less during high volatility states. During corporations usually change their objectives according to the market conditions. For example, SRI firms tend focus high volatility states, SRI firms with the increasing survival and competitive stress will have to shift some of their attention from social goals to financial objectives. Second, for the same reason, SRI firms tend to involve in socially responsible innovations more during low volatility states and less in high volatility states, if they do involve in socially responsible activities. Therefore, the SRI unsystematic risk tends to increase, when the market volatility increases.

Also, this phenomenon can be explained at the portfolio level. First, when the market dives, social screening enables SRI index providers to select firms that have performed financially well. Second, social screening provides flexibility to the SRI indices suppliers to add more weights on some preferable industries, while avoid poorly performed industries. These facts enlarge the asset allocation inequality; and therefore, increase unsystematic risk. Also, the rising unsystematic risk may come from the fact that some social investors withdraw their investments from certain industry sectors or companies of SRI, during the down market.

\subsection{The Relationship between Unsystematic Risk and Return}

Table 5 presents the empirical results from the regression model for the relationship between the unsystematic risk and return during different market regimes, as well as the whole study period. 
Table 5: $\quad$ Empirical Coefficients of Unsystematic Risk

\begin{tabular}{|c|c|c|c|c|c|c|c|}
\hline & DSI & CAL & USSA & USSL & USSM & USSS & FUS \\
\hline$\mu_{1}$ & 0.34 & -1.37 & -0.11 & -0.48 & -1.32 & -0.73 & -2.05 \\
& $(0.8267)$ & $(0.3621)$ & $(0.9493)$ & $(0.7263)$ & $(0.3463)$ & $(0.7318)$ & $(0.2557)$ \\
\hline$\beta_{1}$ & 0.94 & 2.67 & 1.57 & 1.74 & $3.21 *$ & 6.81 & 2.97 \\
& $(0.6897)$ & $(0.2323)$ & $(0.6377)$ & $(0.4132)$ & $(0.0864)$ & $(0.4143)$ & $(0.1538)$ \\
\hline$R^{2}{ }_{1}$ & $0.37 \%$ & $2.45 \%$ & $0.40 \%$ & $1.16 \%$ & $5.07 \%$ & $1.11 \%$ & $3.60 \%$ \\
\hline$\mu_{2}$ & -0.74 & $-8.39 *$ & -8.47 & -7.06 & 1.68 & -2.92 & $-6.67 *$ \\
& $(0.8445)$ & $(0.0969)$ & $(0.1437)$ & $(0.3359)$ & $(0.7325)$ & $(0.5499)$ & $(0.0602)$ \\
\hline$\beta_{2}$ & 7.13 & $9.50^{* *}$ & $16.35^{*}$ & 10.92 & 1.20 & 10.24 & $7.52^{* * *}$ \\
& $(0.1735)$ & $(0.0381)$ & $(0.0545)$ & $(0.2103)$ & $(0.8477)$ & $(0.2574)$ & $(0.0150)$ \\
\hline$R^{2}{ }_{2}$ & $9.06 \%$ & $31.13 \%$ & $23.93 \%$ & $12.74 \%$ & $0.43 \%$ & $12.61 \%$ & $37.63 \%$ \\
\hline$\mu_{3}$ & 1.85 & -2.24 & -6.87 & -9.17 & 5.33 & -6.62 & 0.72 \\
& $(0.6282)$ & $(0.8427)$ & $(0.4958)$ & $(0.5269)$ & $(0.5074)$ & $(0.2924)$ & $(0.9365)$ \\
\hline$\beta_{3}$ & $-9.08 *$ & -0.54 & 4.96 & 7.72 & -8.30 & 5.69 & -2.38 \\
& $(0.0709)$ & $(0.9531)$ & $(0.7471)$ & $(0.6603)$ & $(0.3645)$ & $(0.5223)$ & $(0.7064)$ \\
\hline$R^{2}{ }_{3}$ & $14.70 \%$ & $0.03 \%$ & $0.77 \%$ & $1.42 \%$ & $4.59 \%$ & $2.98 \%$ & $0.97 \%$ \\
\hline$\mu_{f}$ & 3.48 & 0.13 & -1.90 & -0.79 & 1.13 & 0.30 & 0.51 \\
& $(0.1196)$ & $(0.9327)$ & $(0.3851)$ & $(0.6998)$ & $(0.5490)$ & $(0.8071)$ & $(0.7567)$ \\
\hline$\beta_{f}$ & -4.68 & -0.05 & 3.84 & 1.46 & -0.74 & 0.32 & -0.35 \\
& $(0.1367)$ & $(0.9757)$ & $(0.3039)$ & $(0.6101)$ & $(0.7560)$ & $(0.9119)$ & $(0.8219)$ \\
\hline$R_{f}^{2}$ & $2.50 \%$ & $0.00 \%$ & $1.20 \%$ & $0.30 \%$ & $0.11 \%$ & $0.01 \%$ & $0.06 \%$ \\
\hline
\end{tabular}

Notes: 1.The Table is organized as parameters in Regime 1,2,3 and the whole period of forecasting respectively.

2. $\mu, \beta$ and $\mathrm{R}^{2}$ stand for the alpha, Beta coefficient and $\mathrm{R}$ square respectively.

3.Asterisks $*$ and $* *$ denote $\mathrm{H}_{0}$ rejected at a significance level of $10 \%$ and $5 \%$, respectively.

The results in Table 5 suggest that during Regime 1, the Beta coefficients are all positive for the seven SRI indices. However, only the index USSM is statistically significant with a p-value of 8.64 per cent. During Regime 2, the Beta coefficients for most of the seven indices have increased and remained positive. Two indices are significant at 5 per cent level of significance, and three at 10 per cent level. The model $\mathrm{R}$ square for the regressions has increased for all of the seven indices from average 2.02 per cent in Regime 1 to 18.22 per cent in Regime 2. This suggests that social screening does reward investors during low volatility states, and this effect is much more intensified when the market leaps. Our results are in line with the findings of Tang and Shum (2007) and Angelidis and Tessaromatis (2009), which find that unsystematic risks only exist in low volatility and high return market states. During Regime 3, four SRI indices have a negative Beta, and only one is statistically significant at 10 per cent level of significance. This implies that social effect impacts index returns in a negative way during highly volatile market states, although it may not be economically significant.

Table 5 also provides evidence on the forecasting capacity of the unsystematic risk to the indices' returns for the whole period. We find that the Beta coefficients are either positive or negative, but insignificant. This finding indicates that the SRI unsystematic risk has no predictive power for the future returns, based on the time series data over this study period. These results coincide with Bali and Cakici (2008), which conclude that unsystematic risk has no predictive power for the future returns. This evidence further proves that the relationship between SRI return and unsystematic risk is not constant or linear, and the use of MS model is appropriate for the SRI time series.

\section{Conclusion}

In this paper we provide evidence on the importance of unsystematic risk on the SRI return. For the first time, we apply the Markov Switching model to identify the impact of stock market regimes on SRI unsystematic risk-return relationship. 
Utilizing monthly data of seven US SRI equity indices and their corresponding benchmark indices over the period June 2001 to December 2009, we find that unsystematic risk moves in line with total risk. Also, we uncover a positive relationship between unsystematic risk and SRI returns during low and medium volatility states, but not in high volatility states. Our results are consistent with the findings of Tang and Shum (2007) and Angelidis and Tessaromatis (2009) in the sense of the unsystematic risk premium under different market conditions. Moreover, we show that SRI unsystematic risk has no predictive power for future returns. This result is similar to the findings of Bali and Cakici (2008), which concluded the same in relation to the predictive power of the unsystematic risk.

In general, our findings have important implications for the existing or potential investors and institutions in deciding of the time and extent of involving in the SRI activities. The significant positive relationship during the low and medium volatility regimes implies that SRI investors are rewarded for bearing the additional unsystematic risk (idiosyncratic risk) during these market conditions. The insignificant relationship during the high volatility regime indicates that it is uncertain whether the SRI investors are compensated for the unsystematic risk they are bearing when the market dives.

\section{References}

[1] Aaker, D. A., and R. Jacobson, 1987. "The role of risk in explaining differences in profitability", Academy of Management Journal, 30, p. 277.

[2] Angelidis, T., and N. Tessaromatis, 2008. "Does idiosyncratic risk matter? Evidence from European stock markets", Applied Financial Economics, 18, p. 125.

[3] Angelidis, T., and N. Tessaromatis, 2009. "Idiosyncratic risk matters! A regime switching approach", International Review of Economics and Finance, 18, p.132.

[4] Bali, G. T., N., Cakici, X., Yan, and Z., Zhang, 2005. "Does idiosyncratic risk really matter?" The Journal of Finance, 60, p.905.

[5] Bali, T., and N., Cakici, 2008. "Idiosyncratic volatility and the cross section of expected returns", Journal of Financial and Quantitative Analysis, 43, p.29.

[6] Barber, J. R., 1994. "Mutual fund risk measurement and future returns", Quarterly Journal of Business and Economics, 33, p.55.

[7] Bollen, B., A., Skotnicki, A., and M., Veeraraghavan, 2009. "Idiosyncratic volatility and security returns: Australian evidence", Applied Financial Economics, 19, p. 1573.

[8] Carroll, C., and K.C.J., Wei, 1988. "Risk, return, and equilibrium: an extension", The Journal of Business, 6, p.485.

[9] Cheung, Y.-L., and K.T., Wong, 1992. "An assessment of risk and return: Some empirical findings from the Hong Kong stock exchange", Applied Financial Economics, 2, p.105.

[10] Cox, L. A., and G.L., Griepentrog, 1988. "Systematic risk, unsystematic risk, and propertyliability rate regulation", Journal of Risk and Insurance, 55, p.606.

[11] Fu, F., 2005. "Idiosyncratic risk and the cross-section of expected stock returns", Working paper, University of Rochester

[12] Galema, R., A., Plantinga, and B., Scholtens, 2008. "The stocks at stake: return and risk in socially responsible investment", Journal of Banking and Finance, 32, pp.2646-2654.

[13] Gonzalez L., P., Hoang, J., Powell and J., Shi, 2006. "Defining and dating bull and Bear markets: two centuries of evidence", Multinational Finance Journal, 10, p.81.

[14] Goyal, A. and P., Santa-Clara, 2003. "Idiosyncratic risk matters!" The Journal of Finance, 58, p.975.

[15] Guidolin M., and S., Hyde, 2009. "What tames the Celtic tiger? Portfolio implications from a multivariate Markov Switching model”, Applied Financial Economics, 19, p.463.

[16] Jiang, X. and B.S., Lee, 2006. "The dynamic relation between returns and idiosyncratic volatility", Financial Management, 35, p.43. 
[17] Krolzig, H., 1997. "Markov-switching vector auto-regressions”, Springer-Vorlage, Berlin

[18] Merton, R.C., 1987. "A simple model of capital market equilibrium with incomplete information", The Journal of Financem, 42, p.483.

[19] Payaslioglu, C., 2008. "Revisiting East Asian exchange rates: the same spirit under a different sky", Applied Financial Economics, 18, p.1263.

[20] Roca, E., and V., Wong, 2008. "An analysis of the sensitivity of Australian superannuation funds to market movements: a Markov regime switching approach", Applied Financial Economics, 18, p.583.

[21] Schröder, M., 2007. "Is there a difference? The performance characteristics of SRI equity indices", Journal of Business Finance and Accounting, 34, p.331.DD

[22] Statman, M. and D., Glushkov, 2009. "The wages of social responsibility", Financial Analysts Journal, 65, p.33.

[23] Sylvain, S., 2009. "Adaptive posterior mode estimation of a sparse sequence for Model Selection", Scandinavian Journal of Statistics, 36, p.577.

[24] Tang, Y.N.G., and W.C., Shum, 2006. "Risk-return relationships in the Hong Kong stock market: revisit", Applied Financial Economics, 16, p.1047.

[25] Tang, Y.N.G., and W.C., Shum, 2007. "The risk-return relations: evidence from the Korean and Taiwan stock markets", Applied Economics, 39, p.1905. 